\title{
Isorhynchophylline, a Potent Plant Alkaloid, Induces Apoptotic and Anti-Metastatic Effects in Human Hepatocellular Carcinoma Cells through the Modulation of Diverse Cell Signaling Cascades
}

\author{
Hanwool Lee ${ }^{1}$, Seung Ho Baek ${ }^{1,2}$, Jong Hyun Lee ${ }^{1}$, Chulwon Kim ${ }^{1}$, Jeong-Hyeon Ko ${ }^{1}$, \\ Seok-Geun Lee ${ }^{1}$, Arunachalam Chinnathambi ${ }^{3}$, Sulaiman Ali Alharbi ${ }^{3}$, Woong Mo Yang ${ }^{1}$, \\ Jae-Young Um ${ }^{1}$, Gautam Sethi ${ }^{3,4,5, *}$ and Kwang Seok Ahn ${ }^{1, *}$ \\ 1 College of Korean Medicine, Kyung Hee University, 24 Kyungheedae-ro, Dongdaemun-gu, Seoul 02447, \\ Korea; okhwko@naver.com (H.L.); baeksh@woosuk.ac.kr (S.H.B.); 88milkyway@hanmail.net (J.H.L.); \\ sunny10526@nate.com (C.K.); gokjh1647@gmail.com (J.-H.K.); seokgeun@khu.ac.kr (S.-G.L.); \\ wmyang@khu.ac.kr (W.M.Y.); jyum@khu.ac.kr (J.-Y.U.) \\ 2 College of Korean Medicine, Woosuk University, 46 Eoeun-ro, Wansan-gu, Jeonju-si, Jeollabuk-do 54987, Korea \\ 3 Department of Botany and Microbiology, College of Science, King Saud University, Riyadh 11451, \\ Saudi Arabia; dr.arunmicro@gmail.com (A.C.); sharbi@ksu.edu.sa (S.A.A.) \\ 4 School of Biomedical Sciences, Curtin Health Innovation Research Institute, Curtin University, \\ Perth, WA 6009, Australia \\ 5 Department of Pharmacology, Yong Loo Lin School of Medicine, National University of Singapore, \\ Singapore 117600, Singapore \\ * Correspondences: phcgs@nus.edu.sg (G.S.); ksahn@khu.ac.kr (K.S.A.); \\ Tel.: +65-65-163-267 (G.S.); +82-2-961-2316 (K.S.A.); Fax: +65-68-737-690 (G.S.)
}

Academic Editors: Maurizio Battino and Francesca Giampieri

Received: 3 April 2017; Accepted: 16 May 2017; Published: 19 May 2017

\begin{abstract}
Isorhynchophylline (Rhy) is an active pharmacological component of Uncaria rhynchophylla that has been reported previously to exert significant antihypertensive and neuroprotective effects. However, very little is known about its potential anti-cancer activities. This study was carried out to evaluate the anticancer effects of Rhy against various human carcinoma cell lines. We found that Rhy exhibited substantial cytotoxic effect against human hepatocellular carcinoma HepG2 cells when compared with other human carcinoma cell lines including those of lung, pancreas, prostate, head and neck, breast, multiple myeloma, brain and renal cell carcinoma. Rhy induced apoptosis as characterized by accumulation of cells in sub G1 phase; positive Annexin V binding; activation of caspase-8, -9, and -3; and cleavage of PARP (poly-ADP ribose polymerase). This effect of Rhy correlated with the down-regulation of various proteins that mediated cell proliferation, cell survival, metastasis, and angiogenesis. Moreover, cell proliferation, migration, and constitutive CXCR4 (C-X-C chemokine receptor type 4), MMP-9 (Matrix metallopeptidase-9), and MMP-2 expression were inhibited upon Rhy treatment. We further investigated the effect of Rhy on the oncogenic cell signaling cascades through phospho-kinase array profiling assay. Rhy was found to abrogate phospho-p38, ERK, JNK, CREB, c-Jun, Akt, and STAT3 signals, but interestingly enhanced phospho-p53 signal. Overall, our results indicate, for the first time, that Rhy could exert anticancer and anti-metastatic effects through regulation of multiple signaling cascades in hepatocellular carcinoma cells.
\end{abstract}

Keywords: isorhynchophylline; apoptosis; invasion; migration; cell signaling 


\section{Introduction}

Isorhynchophylline (Rhy) is one of the major oxindole alkaloids isolated from Uncaria rhynchophylla, which has already been extensively used for the treatment of asthma, cancer, cirrhosis, diabetes, hypertension, stroke and rheumatism in tropical regions, such as Southeast Asia, Africa and southeast America [1]. Previous studies have demonstrated that Rhy mainly acts on cardiovascular and central nervous system diseases including hypertension, brachycardia, arrhythmia, sedation, vascular dementia, and amnesia [2]. Recently, Kaiser et al. have elegantly evaluated the potential effect of pentacyclic oxindole alkaloids isolated from Uncaria tomentosa on genotoxicity and cytotoxicity against human leukocytes, human bladder cancer cell line (T24) and human glioblastoma cell line (U-251-MG) and found that diverse chemotypes exhibited differential selectivity against human malignant cells [3]. Rhy has also been reported to exhibit anti-inflammatory activities in mouse microglial cells $[4,5]$. However, no reports have been published so far on the anticancer potential of Rhy, and possible molecular mechanism(s) underlying its anticancer effects.

Natural products play an important role in the process of anticancer drug discovery. Because of pharmacological safety, plant-derived natural products as well as their semisynthetic and synthetic analogs contribute significantly to the process of development of novel anti-neoplastic agents $[6,7]$. For a long time, deregulation in the process of apoptosis has been a significant cause of carcinogenesis [8]. Now it is generally agreed that during the formation of cancer the suppression of apoptotic signals could have a very significant effect [9]. Triggering apoptosis in cancer cells has thus become an important method of enhancing the results of therapy during the treatment of cancer. Much evidence has demonstrated that several phytochemicals exert anti-tumorigenic activities by several processes, including preventing the activation of pro-carcinogens, inhibiting cell proliferation, invasion, and angiogenesis, and stimulating sustained apoptosis in tumor cells [10]. A number of dietary agents derived from natural sources can also regulate mitochondrial biogenesis and also simultaneously target various signaling molecules implicated in the apoptotic pathway [11,12]. For example, triptolide, a major active ingredient extracted from the widely used Chinese herb Tripterygium wilfordii Hook $\mathrm{f}$. that has been extensively analyzed for its anticancer effects was reported to induce pathological changes of heart tissue and exhibit cardiotoxicity through the modulation of the mitochondria-mediated apoptotic signaling pathway [13].

The purpose of this study was to investigate the potential anticancer effects of Rhy and elucidate its underlying molecular mechanisms. We particularly aimed to determine the effect of Rhy on the induction of apoptosis and inhibition of metastatic activity in tumor cells. In our experiments, Rhy was found to substantially downregulate the expression of several anti-apoptotic, proliferative, metastatic, and angiogenic gene products, leading to the induction of apoptosis through caspase- $8,-9$, and -3 activation, and also inhibited migratory and invasive potential of tumor cells.

\section{Results}

\subsection{Rhy Suppressed the Cell Viability in Variety of Tumor Cells}

The structure of Rhy has been shown in Figure 1A. To examine the anti-tumor activity of Rhy, HepG2, A549, BxPC-3, Caki-1, RPMI-8226, 786-O, Du145, FaDu, H1299, MDA-MB-231, U266, H4, U87MG, T98G, LN18 and IM-PHFA cells were treated with Rhy $(0,50,100,150,200$, or $300 \mu \mathrm{M})$ for 48 h, and then cell viability was measured by MTT assay. As shown in Figure 1B, Rhy exhibited greatest cytotoxicity against HepG2 cells as compared to other tumor cells as well as immortalized primary human fetal astrocytes (IM-PHFA). 
A.

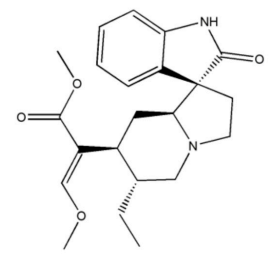

Isorhynchophylline (Rhy)

[MW : 348]

B.
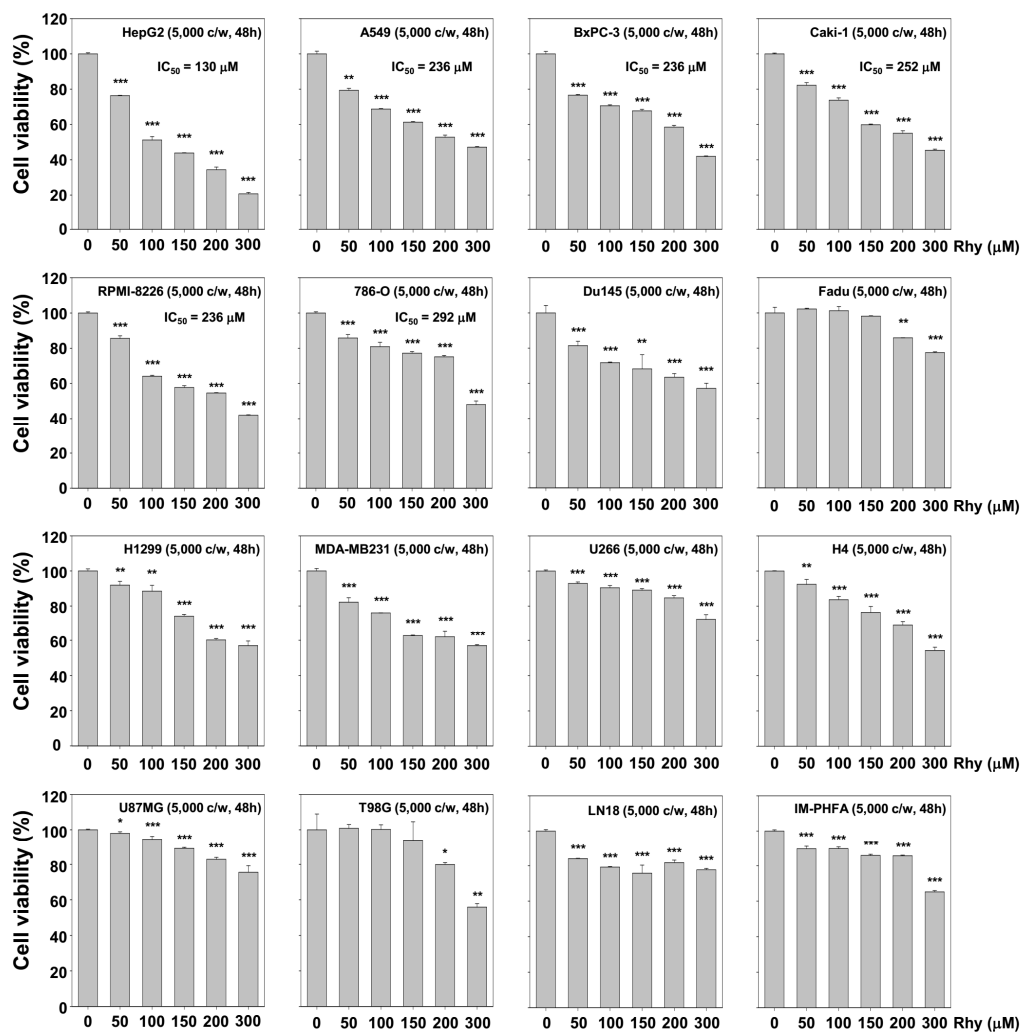

Figure 1. Cytotoxicity of isorhynchophylline (Rhy) on various cell lines: (A) Structure of Rhy; and (B) HepG2, A549, BxPC-3, Caki-1, RPMI-8226, 786-O, Du145, FaDu, H1299, MDA-MB-231, U266, H4, U87MG, T98G, LN18, and IM-PHFA cells were treated with Rhy $(0,50,100,150,200$, or $300 \mu \mathrm{M})$ for $48 \mathrm{~h}$. Values represent the mean $\pm \mathrm{SD}$ of triplicate cultures $\left({ }^{*} p<0.05,{ }^{* *} p<0.01,{ }^{* * *} p<0.001\right)$. Cell viability was analyzed by the MTT method as described under Materials and Methods.

2.2. Rhy Repressed the Expression of Various Proteins Involved in Anti-Apoptosis, Proliferation, Metastasis and Angiogenesis

We next examined the effects of Rhy on the expression of various proteins involved in anti-apoptosis, proliferation, metastasis and angiogenesis in HepG2 cells. As depicted in Figure 2A Rhy suppressed the expression of anti-apoptotic gene products such as Bcl-2 (B-cell lymphoma-2), Bcl-xL (B-cell lymphoma-extra large), Survivin, IAP-1 (inhibitors of apoptosis-1) and IAP-2 (inhibitors of apoptosis-2) in a time-dependent manner. Figure 2B shows that Rhy also substantially repressed the expression of proteins linked with metastasis and angiogenesis including COX-2 (cyclooxygenase-2), 
VEGF (vascular endothelial growth factor), MMP-9, and MMP-2 and cell cycle regulatory protein such as Cyclin D1.

A.

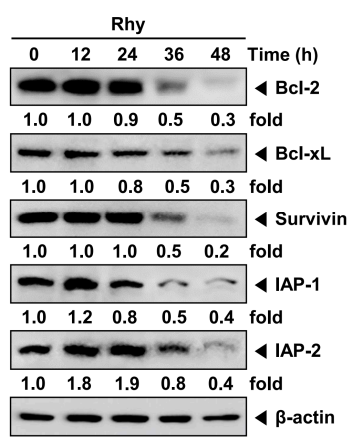

C.

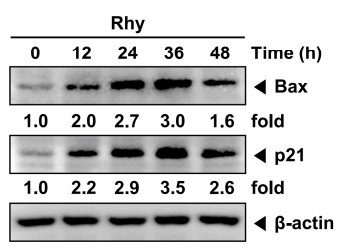

E.

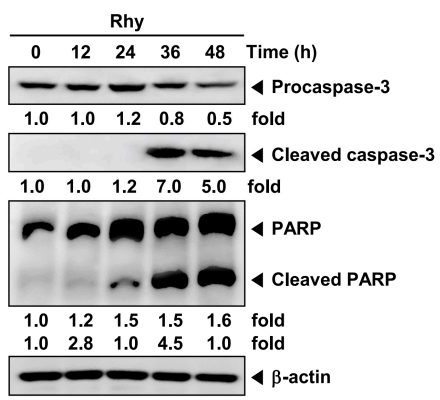

B.

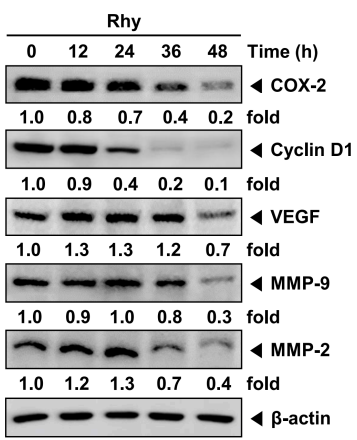

D.

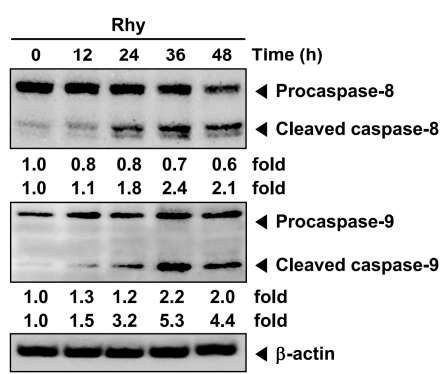

F. NT PEGFP $\begin{gathered}\text { PEGFP. } \\ \text { Bcl-2 }\end{gathered}$

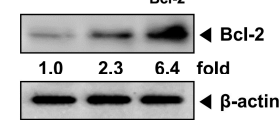

G.

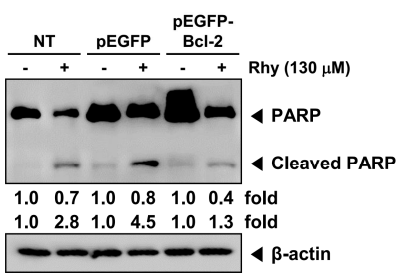

Figure 2. Induction of apoptosis by Rhy in HepG2 cells. (A-C) Rhy modulates expression of various proteins involved in anti-apoptosis, proliferation, angiogenesis, metastasis, and pro-apoptosis. HepG2 cells $\left(1 \times 10^{6}\right.$ cells / well $)$ were incubated with Rhy $(130 \mu \mathrm{M})$ for $0,12,24,36$, and $48 \mathrm{~h}$. Whole-cell extracts were prepared, and $20 \mu \mathrm{g}$ of the whole-cell lysate was resolved by sodium dodecyl sulfate polyacrylamide gel electrophoresis (SDS-PAGE), electrotransferred to nitrocellulose membrane, sliced from the membrane based on the molecular weight, and then probed with antibodies against Bcl-2, Bcl-xL, survivin, inhibitors of apoptosis protein (IAP)-1/2, COX-2, Cyclin D1, VEGF, MMP-9/2, Bax, and p21 as described in the Materials and Methods. The same blots were stripped and reprobed with $\beta$-actin antibody to verify equal protein loading; (D,E) HepG2 cells $\left(1 \times 10^{6}\right.$ cells/well) were treated with Rhy $(130 \mu \mathrm{M})$ for $0,12,14,36$, and $48 \mathrm{~h}$. Thereafter, equal amounts of lysates were analyzed by Western blotting analysis using antibodies against caspase-8/9/3 and PARP. The same blots were stripped and reprobed with $\beta$-actin antibody to verify equal protein loading; (F) Cells were transiently transfected with $\mathrm{pEGFP-Bcl-2}$ or $\mathrm{pEGFP}$ (control vector) plasmid. Bcl-2 protein was overexpressed in pEGFP-Bcl-2 transfected HepG2 cells compared with control. The results shown are representative of the three independent experiments; (G) Transiently transfected cells were treated with Rhy for $48 \mathrm{~h}$. Thereafter, equal amounts of lysate were analyzed by Western blot analysis using antibodies against PARP and $\beta$-actin. 


\subsection{Rhy Induced the Expression of Bax and 221 Proteins}

The Bcl-2 family proteins have emerged as critical regulators of the mitochondria mediated apoptosis by functioning as either promoters (e.g., Bax (Bcl-2-associated X) and Bak (Bcl-2 homologous antagonist/killer)) or inhibitors (e.g., Bcl-2 and Bcl-xL) of the cell death process [14]. Once activated, Bax permeabilizes the mitochondrial outer membrane, resulting in the release of cytochrome $c$ and other pro-apoptotic factors that can induce caspase activation and programmed cell death [15]. Besides, the cyclin-dependent kinase (CDK) inhibitor p21 is a prototypical member of the CIP/KIP family of CDK inhibitors. It negatively modulates cell cycle progression by inhibiting the activities of cyclin D/CDK4 complexes and can block DNA replication by binding to proliferating cell nuclear antigen [16]. We found that Rhy induced the expression of Bax and p21 proteins in a time-dependent manner in HepG2 cells (Figure 2C).

\subsection{Rhy Activated Caspase-3 and Caused PARP Cleavage}

We also evaluated the effect of Rhy on the apoptotic caspase-PARP axis in HepG2 cells. Rhy induced the cleavage of procaspase- 8 and procaspase- 9 as seen by the appearance of its cleavage products (Figure 2D). As shown in Figure 2E, Rhy also induced a time-dependent activation of caspase-3. Activation of caspase-3 led to the cleavage of a $116 \mathrm{kDa}$ PARP (poly-ADP ribose polymerase) protein into $87 \mathrm{kDa}$ fragments. These results clearly suggest that Rhy can induce caspase-3-dependent apoptosis in HepG2 cells.

\subsection{Overexpression of Bcl-2 Attenuated Rhy-Mediated Apoptosis}

We next investigated whether overexpression of Bcl-2 by pEGFP-Bcl-2 plasmid can prevent the observed effects of Rhy on apoptosis. The cells transfected with pEGFP-Bcl-2 clearly showed overexpression of Bcl-2 as compared with those transfected with only control plasmid (Figure 2F). The same blots were stripped and reprobed with $\beta$-actin antibody to verify equal protein loading. As shown in Figure 2G, overexpression of Bcl-2 led to the attenuation of Rhy-mediated cleavage of PARP as compared with the control cells.

\subsection{Rhy Caused the Accumulation of the Cells in the Sub-G1 Phase}

We determined the effect of Rhy on cell cycle distribution in HepG2 cells. Figure 3A shows that Rhy increased the cell accumulation in the sub-G1 phase, which is indicative of apoptosis as compared with the non-treated (NT) cells (Figure 3A).

A.

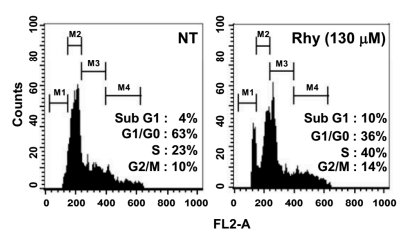

c.

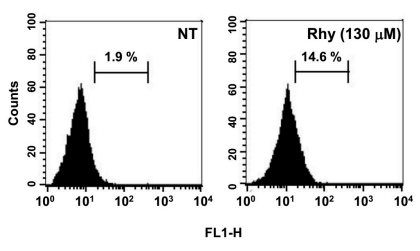

B.

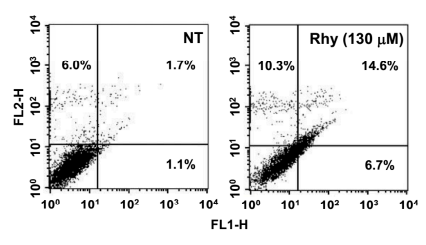

D.
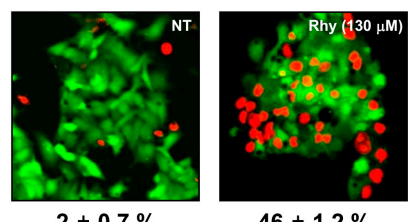

$46 \pm 1.2 \%$

Figure 3. Cont. 
E.

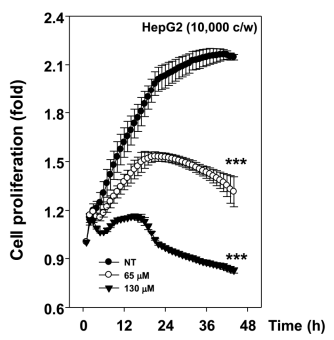

G.

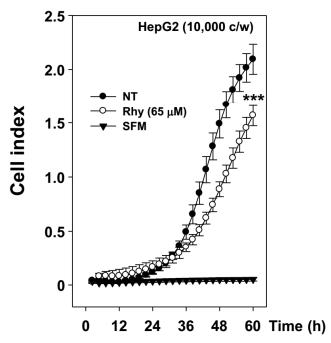

F.

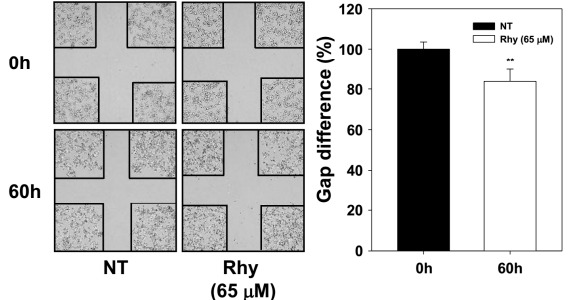

H.

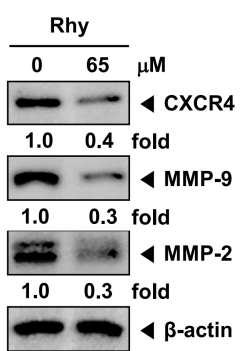

Figure 3. Apoptotic and anti-metastatic effect of Rhy in HepG2 cells. (A) After HepG2 cells $\left(1 \times 10^{6}\right.$ cells/well $)$ were seeded onto six-well plates, they were treated with $130 \mu \mathrm{M}$ of Rhy for $36 \mathrm{~h}$. Then, the cells were harvested, washed with a cold PBS buffer, and digested with RNase. Cellular DNA staining with PI and flow cytometric analysis was done to determine the cell cycle distribution as described in the Materials and Methods; $(\mathbf{B})$ HepG2 cells $\left(1 \times 10^{6}\right.$ cells/well) were treated with Rhy for $48 \mathrm{~h}$. The cells were incubated with an FITC-conjugated Annexin V antibody and then analyzed by a flow cytometry as described in Materials and Methods. Data are representative of the best quality data collected from three individual experiments with similar results and the percentage of late apoptotic cells (upper right quadrant); (C) After HepG2 cells $\left(1 \times 10^{6}\right.$ cells/well) were seeded onto six-well plates, they were treated with $130 \mu \mathrm{M}$ of Rhy for $48 \mathrm{~h}$. The cells were fixed and incubated using TUNEL reaction solution and then analyzed by a flow cytometry. Data are representative of the best quality data collected from three individual experiments with similar results; (D) HepG2 cells were treated with Rhy $(130 \mu \mathrm{M})$ for $36 \mathrm{~h}$. Cells were stained with a live/dead assay reagent for $30 \mathrm{~min}$ and then analyzed under a fluorescence microscope $(40 \times)$ as described in Materials and Methods. Percentage of apoptosis is indicated in the inset; (E) HepG2 cells were plated in triplicate and treated with Rhy ( 65 or $130 \mu \mathrm{M})$, then cell proliferation was measured by real time cell analysis (RTCA). Values represent the mean $\pm \mathrm{SD}$ of triplicate cultures (*** $p<0.001)$; (F) Wound healing assay was performed for evaluating the inhibitory effect of Rhy on HepG2 cells migration. Confluent monolayers of HepG2 cells were scarred, and repair was monitored microscopically after $60 \mathrm{~h}$ of treatment with $65 \mu \mathrm{M}$ Rhy. Width of wound was measured at time 0 and $60 \mathrm{~h}$ of incubation with and without Rhy. Values represent the mean \pm SD of triplicate cultures (** $p<0.01$ ); (G) Invasion assay was performed using the Roche xCELLigence Real-Time Cell Analyzer (RTCA) DP instrument (Roche Diagnostics GmbH, Mannheim, Germany) as described in Materials and Methods. We tested HepG2 cells invasion activity (40,000 cells/well) in the matrigel-coated CIM (cellular invasion/migration)-Plate 16 with Rhy $(65 \mu \mathrm{M}) ;(\mathbf{H})$ Rhy downregulated expression of CXCR4 and MMP-9/2. HepG2 cells $\left(1 \times 10^{6}\right.$ cells/well) were incubated with Rhy $(65 \mu \mathrm{M})$ for $60 \mathrm{~h}$. Whole-cell extracts were prepared, and $20 \mu \mathrm{g}$ of the whole-cell lysate was resolved by sodium dodecyl sulfate polyacrylamide gel electrophoresis (SDS-PAGE), electrotransferred to nitrocellulose membrane, sliced from the membrane based on the molecular weight, and then probed with antibodies against CXCR4, MMP-9, and MMP-2 as described in Materials and Methods. The same blots were stripped and reprobed with $\beta$-actin antibody to verify equal protein loading. FL2-A, gating for single cells based on the area; FL1-H, relative intensity of GFP florescence; NT, Non treat. 


\subsection{Rhy Promoted Substantial Apoptotic Cell Death}

We next examined the effect of Rhy on cellular apoptosis in HepG2 cells. We found that Rhy increased early apoptotic cells in HepG2 cells as observed by the annexin V assay that increased up to $14.6 \%$ as compared with the non-treated cells (1.7\%) (Figure 3B). The induction of apoptosis was further confirmed by TUNEL staining assay. A significant number (up to 14\% of HepG2 cells) stained positively for TUNEL in Rhy treated group as compared with the NT cells (1.9\%) (Figure 3C). The Live and Dead assay (which measures intracellular esterase activity and plasma membrane integrity) shows that treatment of Rhy increases population of apoptotic cells from $2 \%$ to $46 \%$ (Figure 3D)

\subsection{Rhy Suppressed the Proliferative Activity of HepG2 Cells}

To specifically examine the anti-tumor activity of Rhy in HepG2 cells, the cells were treated with Rhy $(65$ and $130 \mu \mathrm{M})$ for indicated time intervals, and then the cell proliferation was analyzed using a real time cell analyzer (RTCA; Roche). As shown in Figure 3E, Rhy suppressed the proliferation in HepG2 cells in a dose-dependent manner.

\subsection{Rhy Suppressed HepG2 Cells Migration and Invasion}

The effect of Rhy on HepG2 cells migration was elucidated, and it was found, using an in vitro wound healing assay, that HepG2 cells migrated slower under the influence of Rhy (Figure 3F). Whether Rhy can modulate HepG2 cells invasion activity was also investigated. To determine this, cells were seeded to the Matrigel (BD Biosciences, San Diego, CA, USA)-coated CIM-Plate 16 with or without Rhy and examined for invasion. As shown in Figure 3G, Rhy significantly suppressed tumor cells invasion activity. We also evaluated the effect of Rhy on the expression of CXCR4, MMP-9, and MMP-2, proteins which play a critical role in tumor metastasis. As shown in Figure $3 \mathrm{H}$, Rhy downregulated the expression of CXCR4, MMP-9, MMP-2 and proteins in HepG2 cells.

\subsection{Rhy Decreased the Phosphorylation Levels of Various Kinase Phosphorylation Sites in HepG2 Cells}

To examine the effects of Rhy on intracellular signaling cascades, we screened the phosphorylation status of multiple cellular kinases in human HepG2, using the human phospho-kinase antibody array kit (Figure 4A,B). Incubation of these membranes with cell lysates can specifically indicate which kinases can be potentially activated in tumor cells and also as whether the same kinases can respond to the various pharmacological inhibitors. Activated p38, ERK, JNK, CREB, c-Jun, Akt, and STAT3 were strongly expressed in non-treated cells. Rhy treated cells showed a decrease in phosphorylated p38, ERK, JNK, CREB, c-Jun, Akt, and STAT3 levels. Phospho-p53 protein was found to be overexpressed upon Rhy treatment. Therefore, in the next experiments, we focused to determine the effect of Rhy on the p38, ERK, JNK, CREB, c-Jun, Akt, STAT3, and p53 signaling pathways in HepG2 cells. 

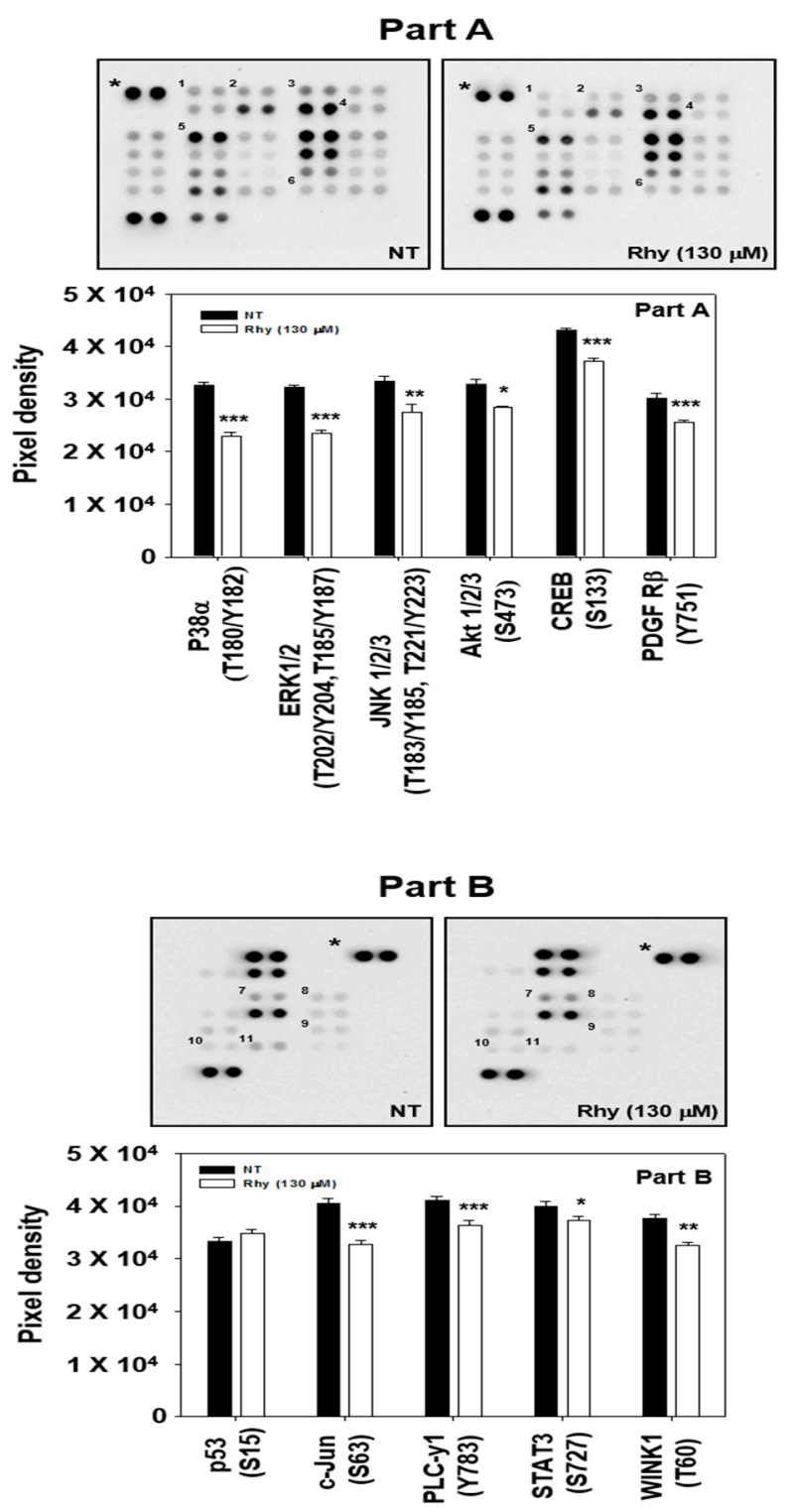

Figure 4. The Human Phospho-Kinase Array detects several phosphorylated proteins in Rhy-treated HepG2 cells. $(\mathbf{A}, \mathbf{B})$ HepG2 cells $\left(1 \times 10^{7}\right.$ cells / well $)$ were incubated with Rhy $(130 \mu \mathrm{M})$ for $12 \mathrm{~h}$. Parts A and B of the array were each incubated with $330 \mu \mathrm{g}$ of cell lysate. Arrays were performed according to the manufacturer's protocols using Human Phospho-Kinase Array Kit (R\&D Systems, Minneapolis, MN, USA), and array images are shown. Array profiles created by quantifying the mean spot pixel densities are shown. Graphs represent spot intensities of indicated proteins. Values represent the mean \pm SD of triplicate cultures $\left({ }^{* *} p<0.001,{ }^{* *} p<0.01,{ }^{*} p<0.05\right)$.

\subsection{Rhy Inhibited Phosphorylation of p38, ERK, JNK, CREB, c-Jun, Akt, STAT3, and Enhanced} Phosphorylation of 553 in HepG2 Cells

Data obtained from our phospho-kinase antibody array studies were further confirmed by Western blotting analysis. Cells were treated with the indicated concentration of Rhy for $12 \mathrm{~h}$. Rhy suppressed the phosphorylation of p38, ERK, JNK, CREB, c-Jun, Akt and STAT3, but enhanced the phosphorylation of p53 in HepG2 cells and had no effect on the expression of total p38, ERK, JNK, CREB, c-Jun, Akt, STAT3, p53 and $\beta$-actin proteins (Figure 5A-D). 
A.

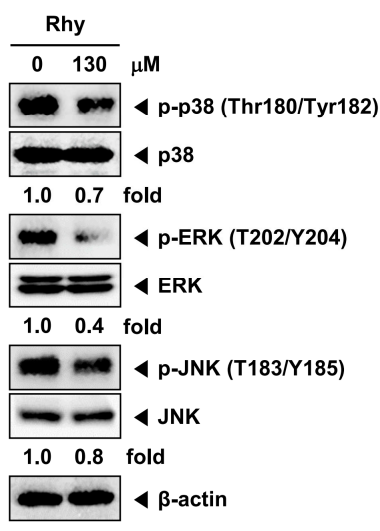

C.

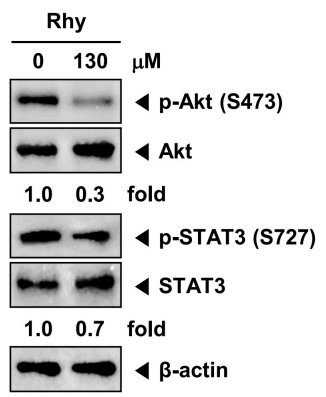

B.

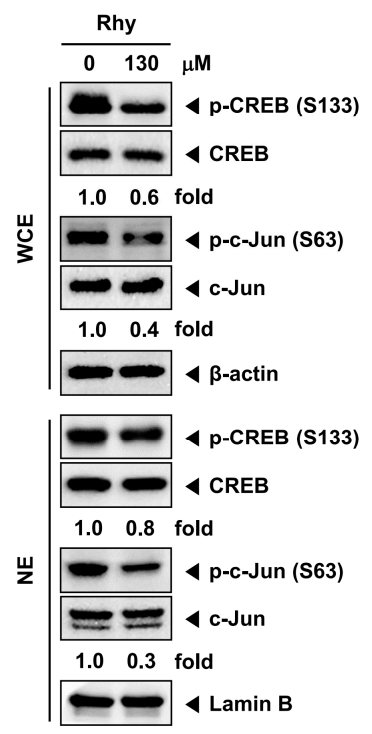

D.

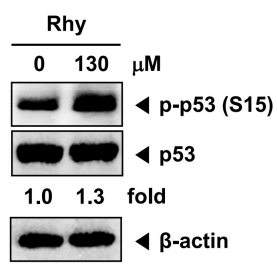

Figure 5. Effect of Rhy on various signal transduction pathways. (A) HepG2 cells $\left(1 \times 10^{6}\right.$ cells/well) were incubated with Rhy $(130 \mu \mathrm{M})$ for $12 \mathrm{~h}$. Whole-cell extracts were prepared, and $20 \mu \mathrm{g}$ of the whole-cell lysate was resolved by sodium dodecyl sulfate polyacrylamide gelelectrophoresis, electrotransferred to nitrocellulose membrane, sliced from the membrane based on the molecular weight, and then probed with antibodies against p-p38, p38, p-ERK, ERK, p-JNK, and JNK as described in Materials and Methods. The same blots were stripped and reprobed with $\beta$-actin antibody to verify equal protein loading. The results shown here are representative of three independent experiments; (B) HepG2 cells $\left(1 \times 10^{6}\right.$ cells /well) were treated with Rhy $(130 \mu \mathrm{M})$ for $12 \mathrm{~h}$. After that, whole-cell extracts and nuclear proteins were extract were equal amounts of lysates were analyzed by Western blot analysis using antibodies against p-CREB, CREB, p-c-Jun, and c-Jun. The same blots were stripped and reprobed with $\beta$-actin and Lamin $B$ antibody to verify equal protein loading. The results shown here are representative of three independent experiments; (C) HepG2 cells $\left(1 \times 10^{6}\right.$ cells/well) were incubated with Rhy $(130 \mu \mathrm{M})$ for $12 \mathrm{~h}$. Whole-cell extracts were prepared, and $20 \mu \mathrm{g}$ of the whole-cell lysate was resolved by sodium dodecyl sulfate polyacrylamide gelelectrophoresis, electrotransferred to nitrocellulose membrane, sliced from the membrane based on the molecular weight, and then probed with antibodies against p-Akt, Akt, p-STAT3, and STAT3 as described in Materials and Methods. The same blots were stripped and reprobed with $\beta$-actin antibody to verify equal protein loading. The results shown here are representative of three independent experiments; (D) HepG2 cells $\left(1 \times 10^{6}\right.$ cells/well) were incubated with Rhy $(130 \mu \mathrm{M})$ for $12 \mathrm{~h}$. Whole-cell extracts were prepared, and $20 \mu \mathrm{g}$ of the whole-cell lysate was resolved by sodium dodecyl sulfate polyacrylamide gelelectrophoresis, electrotransferred to nitrocellulose membrane, sliced from the membrane based on the molecular weight, and then probed with antibodies against p-p53, and p53 as described in Materials and Methods. The same blots were stripped and reprobed with $\beta$-actin antibody to verify equal protein loading. 


\section{Discussion}

The aim of this study was to investigate the anticancer and anti-metastatic activities of Rhy against tumor cells. We found that Rhy exhibited highest cytotoxic activity against human hepatocellular carcinoma HepG2 cells. Therefore, we employed HepG2 cells to investigate the detailed mechanism of action of anticancer effects of Rhy. Interestingly, we found that Rhy downregulated the expression of various genes that suppress apoptosis, mediate proliferation, invasion, and angiogenesis. Rhy also induced the inhibition of proliferation, cell cycle arrest, and apoptosis. We further observed that Rhy inhibited the cell migration, invasion, and constitutive CXCR4, MMP-9, and MMP-2 expression in HepG2 cells. We also noted that Rhy suppressed the phosphorylation of p38, ERK, JNK, CREB, c-Jun, Akt, and STAT3 (signal transducer and activator of transcription 3) and also enhanced the phosphorylation of p53 at Ser15 residue (Figure 6).

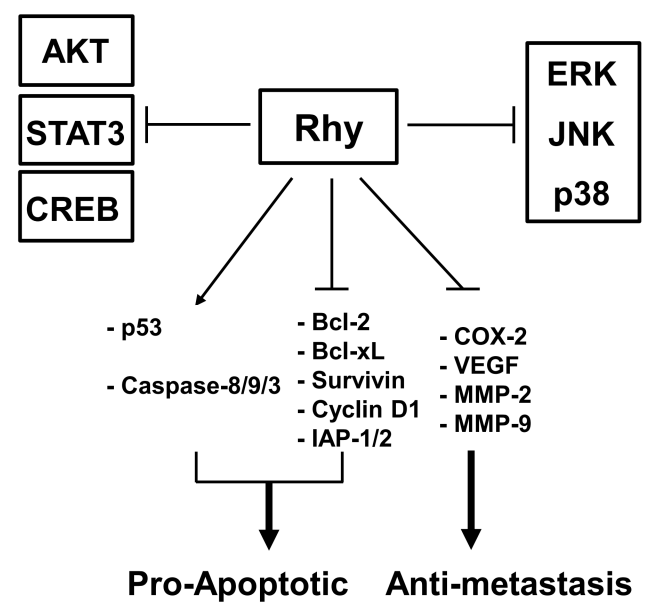

Figure 6. A schematic diagram depicting various molecular targets modulated upon Rhy treatment in tumor cells.

Among genus Uncaria, Uncaria rhynchophylla is the less-investigated species for its anticancer activity. Recently, the aqueous extract of Uncaria rhynchophylla has been reported to be beneficial for the treatment of hypertension, cancer and neurological disorders [17-20]. In this study, we found for the first time that Rhy inhibited the growth of a wide variety of cancer cells. We examined the molecular mechanisms of cancer cell death induced by Rhy. We found that Rhy induced apoptosis through the activation of diverse caspases, including 8, 9, and 3, and caused PARP cleavage. Caspase-3 can be activated by mitochondrial or intrinsic pathway involving caspase- 9 or an extrinsic pathway involving caspase- 8 [21,22]. The results of the present study provide evidence to indicate that Rhy induced apoptosis is partly dependent on the activation of both caspase- 8 and caspase- 9 . We also found that Rhy decreased the expression of anti-apoptotic proteins, Bcl-2, Bcl-xL, survivin, IAP-1, and IAP-2 and increased the expression of pro-apoptotic protein, Bax. Bcl-2 family proteins are localized in mitochondria and regulate caspase activation [23]. Both Bcl-2 and Bcl-xL are known to form heterodimers with another pro-apoptotic member of Bcl-2 family of protein, Bad, and thus can suppress apoptosis [24]. Bax can induce apoptosis through the release of cytochrome $\mathrm{c}$ and induce the activation of caspases [25]. Another apoptosis-regulatory protein family, IAPs, is considered to be an important target to modulate apoptotic cell death in many cancer cells. Inhibitors of apoptosis proteins family proteins regulate apoptosis by binding and inhibiting caspases [26,27]. A variety of prior studies have provided conclusive evidence(s) that elevation in Bcl-2 expression can cause resistance to chemotherapeutic drugs, while decrease in Bcl-2 expression can promote apoptotic responses to anticancer drugs [28]. Our results also indicate that the ectopic expression of Bcl-2 proteins can blocks Rhy-induced apoptosis and this was to be associated with the suppression of PARP cleavage. 
The downregulation of cell cycle promoting cyclin D1 expression and upregulation of cell cycle inhibitor p21 expression by Rhy correlated with the suppression in proliferation and accumulation of cells in the sub-G1 phase of the cell cycle. We also found that Rhy induced the downregulation of pro-angiogenic and metastatic factors COX-2 and VEGF. The CXCR4 chemokine receptor is expressed in a variety of cancers and has been linked to proliferation, invasion, and metastasis in many tumor cells, thus CXCR4 has been suggested as an important therapeutic target for inhibiting cancer metastasis [29]. Additionally, the invasion and metastasis of malignant cells has been found to be closely associated with the degradation of basement membranes and stromal extracellular matrix (ECM) [30]. The matrix metalloproteinases (MMPs), such as MMP-9 and MMP-2, play a critical role in cancer cell invasion and metastasis, which can degrade most components of the ECM [31-35]. We further found that the downregulation of constitutive CXCR4, MMP-9, and MMP-2 expression by Rhy directly correlated with its observed significant anti-invasive effects in the HepG2 cells.

The neuroprotective effect of IRN has been reported to be associated with the enhancement of $\mathrm{p}$-CREB expression mediated via the activation of PI3K/Akt/GSK-3 $\beta$ signaling pathways [36]. Rhy can also contribute to the regulation of MAPKs, NF- $\mathrm{kB}, \mathrm{STAT} 3$, and Akt signal transduction in diverse disease models $[5,36,37]$. In the present study, using phospho-kinase array analysis, we have identified a few important potential targets for Rhy in HepG2 cells. Our studies offer first evidence about the ability of Rhy to suppress the phosphorylation of MAPK, Akt, STAT3 and to enhance the phosphorylation of p53. Mitogen-activated protein kinases (MAPKs) such as ERK, p38 kinase, and JNK pathways regulate cellular proliferation, apoptosis, and differentiation [38]. We show that Rhy inhibited the phosphorylation of ERK, p38, JNK(c-Jun) using both human phospho-antibody array system and Western blot analysis. However, it is still not entirely clear whether the diverse molecular targets modulated by Rhy to exhibit various neuroprotective effects may also play a prominent role in its observed anticancer effects in diverse tumor cell lines.

Recent studies have reported that CREB is involved tumor initiation, progression and metastasis, thereby supporting its role as a proto-oncogene [39]. MAPKs (e.g., p38 and ERK) can phosphorylate and activate CREB-1, thereby promoting the pro-survival signaling that is important for cancer development and progression [40]. We also found that Rhy can substantially suppress the phosphorylation of CREB in HepG2 cells.

Signal transducer and activator of transcription 3 (STAT3) is an oncogenic transcription factor that is constitutively activated in many human cancers. STAT-3 can regulate the expression of genes that mediate survival, proliferation, invasion, and angiogenesis. Several natural chemopreventive agents have been found to be quite effective in suppressing STAT3 activation [41]. STAT3 activation is also tightly regulated by phosphorylation by intrinsic upstream tyrosine kinase and by MAPKs (e.g., p38 and ERK) [42-44]. The suppression of STAT3 phosphorylation by Rhy also correlated with the downregulation of STAT3-regulated gene products in HepG2 cells. These results suggest that STAT3 may be a possible target contributing to the anticancer effects of Rhy.

Akt can regulate a number of downstream target genes which may have pleiotropic effects on both cell survival and proliferation [45], and the deregulation of Akt/p70S6K signal pathways can contribute significantly to the processes of tumorigenesis and metastasis [46,47]. In this study, remarkable reduction of Akt phosphorylation upon Rhy treatment could play a critical role in the inhibition of tumorigenesis.

The p53 tumor suppressor gene is frequently found to be mutated in many human carcinomas [48]. Activation of p53 in response to a variety of stress signals can result in cell death. The role of p53 in apoptosis has been intensely studied for decades [49]. It is known that p53 phosphorylation at the Ser-15 residue is responsible for the activation of apoptotic machinery [50]. We observed that Rhy can increase the phosphorylation of p53 at Ser15 residue using both human phosphor-kinase array and Western blot analysis. The present study has few major limitations. First, most of the in vitro experiments have been predominantly performed in only one tumor cell line namely, HepG2. Second, preclinical therapeutic 
efficacy of Rhy has not been validated under in vivo settings. Lastly, no systematic analysis has been performed related to the toxicity as well as pharmacokinetic profile of Rhy.

Overall, our results indicate that the anticancer and anti-metastatic effects of Rhy in HepG2 cells are mediated through the regulation of various signal transduction cascades and provide a strong rationale for further pursuing the use of Rhy for preclinical validation in appropriate tumor models.

\section{Materials and Methods}

\subsection{Reagents}

Isorhynchophylline (Rhy) was dissolved in dimethyl sulfoxide as a $100 \mathrm{mM}$ stock solution and stored at $-20^{\circ} \mathrm{C}$ and then diluted as needed in cell culture medium. The 3-(4,5-dimethylthiazol-2-yl)2,5-diphenyltetrazolium bromide (MTT), Propidium iodide (PI), Tris base, glycine, $\mathrm{NaCl}$, sodium dodecylsulfate (SDS), bovine serum albumin (BSA), Roswell Park Memoria IIn stitute medium (RPMI) 1640, Minimum Essential Medium (MEM), Dulbecco's Modified Eagle's Medium (DMEM), fetal bovine serum (FBS), were obtained from Thermo Fisher Scientific Inc. (Waltham, MA, USA). Annexin V was purchased from BD Biosciences (Palo Alto, CA, USA). Anti-CXCR4 was obtained from Abcam (Cambridge, MA, USA). Anti-Cyclin D1, anti-cleaved caspase-3, anti-cleaved caspase-8, anti-caspase-8, anti-cleaved caspase-9, anti-caspase-9, anti-p-ERK, anti-ERK, anti-p-p38, anti-p38, anti-p-JNK, anti-JNK, anti-p-Akt, anti-p-STAT3, anti-p-p53, anti-p-CREP , anti-CREB were purchased from Cell Signaling Technology (Beverly, MA, USA). Anti-Bcl-2, anti-Bcl-xl, anti-Survivin, anti-IAP-1, anti-IAP-2, anti-COX-2, anti-VEGF, anti-MMP-9, anti-MMP-2, anti-Bax, anti-p21, anti-caspase-3, anti-PARP, anti-Akt, anti-STAT3, anti-p53, anti-p-c-Jun, anti-c-Jun, anti- $\beta$-actin, and horseradish peroxidase (HRP)-conjugated secondary antibodies were obtained from Santa Cruz Biotechnology (Santa Cruz, CA, USA). Nitrocellulose membrane and nylon membrane were purchased from Pall Corporation (Washington, NY, USA).

\subsection{Cell Lines}

Human various tumor cells including HepG2 (Liver), A549 (Lung), BxPC-3 (Pancreas), Caki-1 (Kidney), RPMI-8226 (Blood), 786-O (Kidney), Du145 (Prostate), FaDu (Head and Neck), H1299 (Lung), MDA-MB-231 (Breast), U266 (Blood), H4 (Brain), U87MG (Brain), T98G (Brain), LN18 (Brain), and IM-PHFA (Brain) cells were obtained from the American Type Culture Collection (Manassas, VA, USA). HepG2, A549, BxPC-3, Caki-1, RPMI-8226, 786-O, Du145, H1299, MDA-MB-231, and U266 cells were cultured in RPMI 1640 medium containing 10\% FBS, penicillin (100 units $/ \mathrm{mL})$, and streptomycin $(100 \mu \mathrm{g} / \mathrm{mL})$. FaDu cells were cultured in MEM containing 10\% FBS, penicillin (100 units $/ \mathrm{mL})$, and streptomycin $(100 \mu \mathrm{g} / \mathrm{mL})$. H4, U87MG, T98G, LN18, and IM-PHFA cells were cultured in DMEM containing 10\% FBS, penicillin (100 units $/ \mathrm{mL})$, and streptomycin $(100 \mu \mathrm{g} / \mathrm{mL})$.

\subsection{MTT Assay}

Cell viability was measured by an MTT assay to detect NADH-dependent dehydrogenase activity. Fifty microliters of MTT solution $(2 \mathrm{mg} / \mathrm{mL})$ in $1 \times$ phosphate-buffered saline (PBS) was directly added to the cells, which were then incubated for $2 \mathrm{~h}$ to allow MTT to metabolize to formazan. Absorbance was measured with an automated spectrophotometric plate reader at a wavelength of $570 \mathrm{~nm}$. Cell viability was normalized as relative percentages in comparison with untreated controls.

\subsection{Western Blot Analysis}

After the cells were treated with the indicated concentrations of Rhy, the HepG2 cells were lysed and the total protein concentrations were determined by Bradford reagent (Bio-Rad, Hercules, CA, USA). Equal amounts of lysates resolved on sodium dodecyl-polyacrylamide gel electrophoresis (SDS-PAGE) were transferred to a nitrocellulose membrane, and the membrane was blocked with $1 \times$ TBS containing $0.1 \%$ Tween 20 and $5 \%$ skim milk at room temperature. After the blocking, the membranes were incubated 
overnight at $4{ }^{\circ} \mathrm{C}$ with the respective primary antibodies. The membranes were washed three times and incubated with diluted horseradish peroxidase (HRP)-conjugated secondary antibodies (1:5000) for $1 \mathrm{~h}$ at room temperature. After four times washing, the membranes were detected using an enhanced chemiluminescence (ECL) kit (Millipore, Bedford, MA, USA).

\subsection{Transfection with $p E G F P-B c l-2$ Plasmids}

We used a commercially available electroporation system, the Neon ${ }^{\mathrm{TM}}$ Transfection System (Invitrogen, Carlsbad, CA, USA) for transfection experiments. HepG2 cells were prepared for transfection after cells were resuspended with $120 \mu \mathrm{L}$ of Neon Resuspension Buffer R for every one million cells. For each electroporation, HepG2 cells with $0.25 \mu \mathrm{g}$ of pEGFPBcl-2 plasmids (provided by Case Western Reserve University) were aliquoted into a sterile microcentrifuge tube. After $48 \mathrm{~h}$ of transfection, HepG2 cells were treated with $130 \mu \mathrm{M}$ of Rhy for $48 \mathrm{~h}$, and whole-cell extracts were prepared for Bcl-2, PARP, and $\beta$-actin analysis by Western blot.

\subsection{Cell Cycle Analysis}

To determine apoptosis, cell cycle analysis was performed using PI. After treatment with $130 \mu \mathrm{M}$ of Rhy for $36 \mathrm{~h}$, the cells were collected, washed with cold PBS, fixed with 70\% ethanol, and incubated for $30 \mathrm{~min}$ at $37^{\circ} \mathrm{C}$ with $0.1 \%$ RNase A in PBS. Cells were then washed, resuspended, and stained in PBS containing $10 \mu \mathrm{g} / \mathrm{mL}$ of PI for $30 \mathrm{~min}$ at room temperature. Cell distribution across the cell cycle was analyzed with a flow cytometry (Becton-Dickinson, Heidelberg, Germany). Acquisition and analysis of the data were performed using Cell Quest 3.0 software (BD Biosciences, Becton-Dickinson, Franklin Lakes, NJ, USA).

\subsection{Annexin V Assay}

One of the early indicators of apoptosis is the rapid translocation and accumulation of the membrane phospholipid phosphatidylserine from the cell's cytoplasmic interface to the extracellular surface. This loss of membrane asymmetry can be detected using the binding properties of Annexin $\mathrm{V}$. To detect apoptosis, we used Annexin $\mathrm{V}$ antibody conjugated with the fluorescent dye fluorescein isothiocyanate (FITC). HepG2 cells were treated with $130 \mu \mathrm{M}$ of Rhy for $36 \mathrm{~h}$ and then subjected to Annexin V and PI staining. The cells were washed and observed accordingly with a flow cytometry (Becton-Dickinson). Acquisition and analysis of the data were performed using Cell Quest 3.0 software.

\subsection{TUNEL Assay}

Late apoptotic cell death was determined using a Roche Diagnosis TUNEL (terminal transferase mediated dUTP-fluorescein nick end labeling) assay kit according to the manufacturer's instructions. Briefly, HepG2 cells treated with $130 \mu \mathrm{M}$ of Rhy for $36 \mathrm{~h}$ and which were washed with cold PBS. The cells were seeded after being fixed with $4 \%$ paraformaldehyde for $30 \mathrm{~min}$ and washed twice with PBS for $2 \mathrm{~min}$. The resuspended cells were put in a permeabilization solution $(0.1 \%$ Triton X-100 and $0.1 \%$ Sodium citrate) at $4{ }^{\circ} \mathrm{C}$ for $20 \mathrm{~min}$, and then the cells were washed with cold PBS. The cells in $45 \mu \mathrm{L}$ of TUNEL enzyme and TUNEL label mixture were incubated for $1 \mathrm{~h}$ at $37^{\circ} \mathrm{C}$ in a humidified atmosphere in the dark. After being washed with PBS, cells were analyzed by a flow cytometery (FACScan Calibur, BD Biosciences, Becton-Dickinson). Acquisition and analysis of the data were performed using Cell Quest 3.0 software.

\subsection{Real-Time Cell Proliferation Analysis}

HepG2 cells (5000 cells/well) were seeded onto 16-well E-plates, integrated with gold microelectrode arrays, then incubated in real-time cell analysis (RTCA) was carried out with the xCELLigence System (Roche, Mannheim, Germany). Application of a low-voltage alternating current signal generates an electric field between the electrodes, which is modulated by the cells covering the 
electrodes. Cell proliferation in the wells results in changes in the impedance readout, obtained from each well with the RTCA DP Instrument. The generated signal is displayed in arbitrary units, referred to as the cell index. After initial incubation on the E-plates for $18 \mathrm{~h}$, HepG2 cells were treated with the Rhy $(65$ and $130 \mu \mathrm{M})$. Non-treated samples were used as controls. The cell index was monitored for $48 \mathrm{~h}$, with measurements every $15 \mathrm{~min}$.

\subsection{Live and Dead Assay}

To measure apoptosis, we used the Live and Dead assay (Invitrogen, Carlsbad, CA, USA), which determines intracellular esterase activity and plasma membrane integrity. This assay employs calcein, a polyanionic dye, which is retained within the live cells and provides green fluorescence. It also employs the ethidium monomer dye (red fluorescence), which can enter the cells only through damaged membranes and bind to nucleic acids but is excluded by the intact plasma membrane of live cells. Briefly, HepG2 cells were seeded at a density of $3 \times 10^{4}$ cells/well in 8-well slide chamber. The cells were incubated with $130 \mu \mathrm{M}$ of Rhy for $36 \mathrm{~h}$. Cells are stained with the Live and Dead reagent ( $5 \mu \mathrm{M}$ ethidium homodimer, $5 \mu \mathrm{M}$ calcein-AM) and then incubated at $37^{\circ} \mathrm{C}$ for $30 \mathrm{~min}$. Cells were analyzed under an Olympus FluoViewFV1000 confocal microscope (Olympus, Tokyo, Japan).

\subsection{Wound Healing Assay}

Before plating the cells, two parallel lines were drawn at the underside of the wells to serve as fiducial marks demarcating the wound areas to be analyzed. Before inflicting the wound, the cells should be fully confluent. The growth medium was aspirated off and replaced by calcium-free phosphate buffered saline to prevent killing of the cells at the edge of the wound by exposure to high calcium concentrations before two parallel scratch wounds were made perpendicular to the marker lines with a sterile $200 \mu \mathrm{L}$ automated pipette tip. Thereafter, the calcium-free medium was then changed to medium with or without $65 \mu \mathrm{M}$ of Rhy. After $60 \mathrm{~h}$, the wounds were observed using bright field microscopy, and multiple images were taken at areas flanking the intersections of the wound and the marker lines at the start and end of the experiment. Gap distance of the wound was measured at three different sites using Photoshop software, and the data were normalized to the average of the control. Graphs were plotted against the percentage of migration distance the cells moved before and after treatment, normalized to control.

\subsection{Invasion Assay}

We employed the Roche xCELLigence Real-Time Cell Analyzer (RTCA) DP instrument (Roche Diagnostics $\mathrm{GmbH}$ ) to measure cellular invasion. The RTCA DP instrument uses the cellular invasion/migration (CIM)-Plate 16, which features microelectronic sensors integrated onto the underside of the microporous polyethylene terephthalate membrane of a Boyden-like chamber. For invasion experiments, the top chamber of the CIM-Plate 16 was coated with Matrigel (BD Biosciences, San Diego, CA, USA) before addition of the medium to the bottom chamber. The CIM-Plate 16 was assembled by placing the top chamber onto the bottom chamber and snapping the two together. Serum-free medium was placed in the top chamber to hydrate and preincubate the membrane for $1 \mathrm{~h}$ in the $\mathrm{CO}_{2}$ incubator at $37^{\circ} \mathrm{C}$ before obtaining a background measurement. Cells were lightly trypsinized, pelleted, and resuspended at the indicated cell densities in serum-freemedium. Once the CIM-Plate 16 has been equilibrated, it was placed in the RTCA DP station, and the background cell-index values were measured. The CIM-Plate 16 was then removed from the RTCA DP station, and the cells are added to the top chamber at the desired density.

\subsection{Human Phospho-Kinase Array}

For antibody arrays, $300 \mu \mathrm{g}$ of cellular extracts were incubated with the Human Phospho-Kinase Array Kit (Proteome Profiler ${ }^{\mathrm{TM}}$; R\&D Systems) following manufacturer's instructions. Densitometry values for antibody array experiments were estimated by the Image 1.6.0 software (National Institutes 
of Health, Bethesda, MA, USA) and were expressed as arbitrary units. Multiple film exposures were used to verify the linearity of the samples analyzed and to avoid saturation of the film. In antibody arrays, the average signal of the pair of duplicate spots, representing each phosphorylated kinase protein, was calculated after subtraction of background values (pixel density) from negative control spots and normalization to average values from positive control spots.

\subsection{Statistical Analysis}

All numeric values are represented as the mean \pm SD. Statistical significance of the data compared with the untreated control was determined using the Student unpaired $t$-test. Significance was set at $p<0.05$.

Acknowledgments: This work was supported by a National Research Foundation of Korea (NRF) grant funded by the Korean government (MSIP) (NRF-2015R1A4A1042399). The authors extend their appreciation to the International Scientific Partnership Program ISPP at King Saud University for funding this research work through ISPP\# 0091.

Author Contributions: Hanwool Lee, Seung Ho Baek, Jong Hyun Lee, Chulwon Kim, and Jeong-Hyeon Ko conceived and designed the study. Hanwool Lee, Seung Ho Baek, Jong Hyun Lee, Chulwon Kim, Jeong-Hyeon Ko, Seok-Geun Lee, Woong Mo Yang, and Jae-Young Um developed the methodology. Seung Ho Baek, Jong Hyun Lee, Arunachalam Chinnathambi, and Sulaiman Ali Alharbi analyzed and interpreted of data. Hanwool Lee, Gautam Sethi, and Kwang Seok Ahn wrote, reviewed, and/or revised the manuscript. Gautam Sethi and Kwang Seok Ahn supervised the study.

Conflicts of Interest: The authors declare no conflict of interest.

\section{References}

1. Zhang, Q.; Zhao, J.J.; Xu, J.; Feng, F.; Qu, W. Medicinal uses, phytochemistry and pharmacology of the genus Uncaria. J. Ethnopharmacol. 2015, 173, 48-80. [CrossRef] [PubMed]

2. Zhou, J.Y.; Zhou, S.W. Isorhynchophylline: A plant alkaloid with therapeutic potential for cardiovascular and central nervous system diseases. Fitoterapia 2012, 83, 617-626. [CrossRef] [PubMed]

3. Kaiser, S.; Carvalho, A.R.; Pittol, V.; Dietrich, F.; Manica, F.; Machado, M.M.; de Oliveira, L.F.; Oliveira Battastini, A.M.; Ortega, G.G. Genotoxicity and cytotoxicity of oxindole alkaloids from Uncaria tomentosa (cat's claw): Chemotype relevance. J. Ethnopharmacol. 2016, 189, 90-98. [CrossRef] [PubMed]

4. Yuan, D.; Ma, B.; Wu, C.; Yang, J.; Zhang, L.; Liu, S.; Wu, L.; Kano, Y. Alkaloids from the leaves of Uncaria rhynchophylla and their inhibitory activity on NO production in lipopolysaccharide-activated microglia. J. Nat. Prod. 2008, 71, 1271-1274. [CrossRef] [PubMed]

5. Yuan, D.; Ma, B.; Yang, J.Y.; Xie, Y.Y.; Wang, L.; Zhang, L.J; Kano, Y.; Wu, C.F. Anti-inflammatory effects of rhynchophylline and isorhynchophylline in mouse $\mathrm{N} 9$ microglial cells and the molecular mechanism. Int. Immunopharmacol. 2009, 9, 1549-1554. [CrossRef] [PubMed]

6. Shanmugam, M.K.; Lee, J.H.; Chai, E.Z.; Kanchi, M.M.; Kar, S.; Arfuso, F.; Dharmarajan, A.; Kumar, A.P.; Ramar, P.S.; Looi, C.Y.; et al. Cancer prevention and therapy through the modulation of transcription factors by bioactive natural compounds. Semin. Cancer Biol. 2016, 40, 35-47. [CrossRef] [PubMed]

7. Bishayee, A.; Sethi, G. Bioactive natural products in cancer prevention and therapy: Progress and promise. Semin. Cancer Biol. 2016, 40, 1-3. [CrossRef] [PubMed]

8. Reed, J.C. Mechanisms of apoptosis. Am. J. Pathol. 2000, 157, 1415-1430. [CrossRef]

9. Igney, F.H.; Krammer, P.H. Death and anti-death: Tumour resistance to apoptosis. Nat. Rev. Cancer 2002, 2, 277-288. [CrossRef] [PubMed]

10. Shanmugam, M.K.; Kannaiyan, R.; Sethi, G. Targeting cell signaling and apoptotic pathways by dietary agents: Role in the prevention and treatment of cancer. Nutr. Cancer 2011, 63, 161-173. [CrossRef] [PubMed]

11. Thushara, R.M.; Hemshekhar, M.; Santhosh, M.S.; Devaraja, S.; Kemparaju, K.; Girish, K.S. Differential action of phytochemicals on platelet apoptosis: A biological overview. Curr. Med. Chem. 2013, 20, 1018-1027. [CrossRef] [PubMed]

12. Forbes-Hernandez, T.Y.; Giampieri, F.; Gasparrini, M.; Mazzoni, L.; Quiles, J.L.; Alvarez-Suarez, J.M.; Battino, M. The effects of bioactive compounds from plant foods on mitochondrial function: A focus on apoptotic mechanisms. Food Chem. Toxicol. 2014, 68, 154-182. [CrossRef] [PubMed] 
13. Zhou, J.; Xi, C.; Wang, W.; Fu, X.; Jinqiang, L.; Qiu, Y.; Jin, J.; Xu, J.; Huang, Z. Triptolide-induced oxidative stress involved with Nrf2 contribute to cardiomyocyte apoptosis through mitochondrial dependent pathways. Toxicol. Lett. 2014, 230, 454-466. [CrossRef] [PubMed]

14. Adams, J.M.; Cory, S. The Bcl-2 protein family: Arbiters of cell survival. Science 1998, 281, $1322-1326$. [CrossRef] [PubMed]

15. Martinou, J.C.; Green, D.R. Breaking the mitochondrial barrier. Nat. Rev. Mol. Cell Biol. 2001, 2, $63-67$. [CrossRef] [PubMed]

16. Luo, Y.; Hurwitz, J.; Massague, J. Cell-cycle inhibition by independent CDK and PCNA binding domains in p21Cip1. Nature 1995, 375, 159-161. [CrossRef] [PubMed]

17. Fujiwara, H.; Iwasaki, K.; Furukawa, K.; Seki, T.; He, M.; Maruyama, M.; Tomita, N.; Kudo, Y.; Higuchi, M.; Saido, T.C.; et al. Uncaria rhynchophylla, a Chinese medicinal herb, has potent antiaggregation effects on Alzheimer's $\beta$-amyloid proteins. J. Neurosci. Res. 2006, 84, 427-433. [CrossRef] [PubMed]

18. Kim, T.J.; Lee, J.H.; Lee, J.J.; Yu, J.Y.; Hwang, B.Y.; Ye, S.K.; Shujuan, L.; Gao, L.; Pyo, M.Y.; Yun, Y.P. Corynoxeine isolated from the hook of Uncaria rhynchophylla inhibits rat aortic vascular smooth muscle cell proliferation through the blocking of extracellular signal regulated kinase $1 / 2$ phosphorylation. Biol. Pharm. Bull. 2008, 31, 2073-2078. [CrossRef] [PubMed]

19. Lee, J.; Son, D.; Lee, P.; Kim, S.Y.; Kim, H.; Kim, C.J.; Lim, E. Alkaloid fraction of Uncaria rhynchophylla protects against $N$-methyl-D-aspartate-induced apoptosis in rat hippocampal slices. Neurosci. Lett. 2003, 348, 51-55. [CrossRef]

20. Lee, J.S.; Kim, J.; Kim, B.Y.; Lee, H.S.; Ahn, J.S.; Chang, Y.S. Inhibition of phospholipase c $\gamma 1$ and cancer cell proliferation by triterpene esters from Uncaria rhynchophylla. J. Nat. Prod. 2000, 63, 753-756. [CrossRef] [PubMed]

21. Thornberry, N.A.; Lazebnik, Y. Caspases: Enemies within. Science 1998, 281, 1312-1316. [CrossRef] [PubMed]

22. Wolf, B.B.; Green, D.R. Suicidal tendencies: Apoptotic cell death by caspase family proteinases. J. Biol. Chem. 1999, 274, 20049-20052. [CrossRef] [PubMed]

23. Zamzami, N.; Brenner, C.; Marzo, I.; Susin, S.A.; Kroemer, G. Subcellular and submitochondrial mode of action of Bcl-2-like oncoproteins. Oncogene 1998, 16, 2265-2282. [CrossRef] [PubMed]

24. Yang, E.; Zha, J.; Jockel, J.; Boise, L.H.; Thompson, C.B.; Korsmeyer, S.J. Bad, a heterodimeric partner for Bcl-XL and Bcl-2, displaces Bax and promotes cell death. Cell 1995, 80, 285-291. [CrossRef]

25. Lin, J.W.; Chen, J.T.; Hong, C.Y.; Lin, Y.L.; Wang, K.T.; Yao, C.J.; Lai, G.M.; Chen, R.M. Honokiol traverses the blood-brain barrier and induces apoptosis of neuroblastoma cells via an intrinsic bax-mitochondrion-cytochrome c-caspase protease pathway. Neuro-Oncology 2012, 14, 302-314. [CrossRef] [PubMed]

26. Richter, B.W.; Duckett, C.S. The IAP proteins: Caspase inhibitors and beyond. Sci. STKE 2000, 2000, pe1. [CrossRef] [PubMed]

27. Yang, Y.L.; Li, X.M. The IAP family: Endogenous caspase inhibitors with multiple biological activities. Cell Res. 2000, 10, 169-177. [CrossRef] [PubMed]

28. Reed, J.C. Bcl-2 family proteins: Regulators of apoptosis and chemoresistance in hematologic malignancies. Semin. Hematol. 1997, 34, 9-19. [PubMed]

29. Proudfoot, A.E. Chemokine receptors: Multifaceted therapeutic targets. Nat. Rev. Immunol. 2002, 2, $106-115$. [CrossRef] [PubMed]

30. Brummer, O.; Athar, S.; Riethdorf, L.; Loning, T.; Herbst, H. Matrix-metalloproteinases 1, 2, and 3 and their tissue inhibitors 1 and 2 in benign and malignant breast lesions: An in situ hybridization study. Virchows Arch. 1999, 435, 566-573. [CrossRef] [PubMed]

31. Liotta, L.A.; Steeg, P.S.; Stetler-Stevenson, W.G. Cancer metastasis and angiogenesis: An imbalance of positive and negative regulation. Cell 1991, 64, 327-336. [CrossRef]

32. Cox, G.; O’Byrne, K.J. Matrix metalloproteinases and cancer. Anticancer Res. 2001, 21, 4207-4219. [PubMed]

33. Egeblad, M.; Werb, Z. New functions for the matrix metalloproteinases in cancer progression. Nat. Rev. Cancer 2002, 2, 161-174. [CrossRef] [PubMed]

34. Xie, T.X.; Wei, D.; Liu, M.; Gao, A.C.; Ali-Osman, F.; Sawaya, R.; Huang, S. STAT3 activation regulates the expression of matrix metalloproteinase-2 and tumor invasion and metastasis. Oncogene 2004, 23, 3550-3560. [CrossRef] [PubMed] 
35. Azzam, H.S.; Arand, G.; Lippman, M.E.; Thompson, E.W. Association of MMP-2 activation potential with metastatic progression in human breast cancer cell lines independent of MMP-2 production. J. Natl. Cancer Inst. 1993, 85, 1758-1764. [CrossRef] [PubMed]

36. Xian, Y.F.; Lin, Z.X.; Mao, Q.Q.; Chen, J.N.; Su, Z.R.; Lai, X.P.; Ip, P.S. Isorhynchophylline Protects PC12 cells against $\beta$-amyloid-induced apoptosis via PI3K/Akt signaling pathway. Evid. Based Complement. Altern. Med. 2013, 2013, 163057. [CrossRef] [PubMed]

37. Guo, H.; Zhang, X.; Cui, Y.; Deng, W.; Xu, D.; Han, H.; Wang, H.; Chen, Y.; Li, Y.; Wu, D.; et al. Isorhynchophylline protects against pulmonary arterial hypertension and suppresses PASMCs proliferation. Biochem. Biophys. Res. Commun. 2014, 450, 729-734. [CrossRef] [PubMed]

38. Reddy, K.B.; Nabha, S.M.; Atanaskova, N. Role of MAP kinase in tumor progression and invasion. Cancer Metastasis Rev. 2003, 22, 395-403. [CrossRef] [PubMed]

39. Xiao, X.; Li, B.X.; Mitton, B.; Ikeda, A.; Sakamoto, K.M. Targeting CREB for cancer therapy: Friend or foe. Curr. Cancer Drug Targets 2010, 10, 384-391. [CrossRef] [PubMed]

40. Swarthout, J.T.; Tyson, D.R.; Jefcoat, S.C., Jr.; Partridge, N.C. Induction of transcriptional activity of the cyclic adenosine monophosphate response element binding protein by parathyroid hormone and epidermal growth factor in osteoblastic cells. J. Bone Miner. Res. 2002, 17, 1401-1407. [CrossRef] [PubMed]

41. Chai, E.Z.; Shanmugam, M.K.; Arfuso, F.; Dharmarajan, A.; Wang, C.; Kumar, A.P.; Samy, R.P.; Lim, L.H.; Wang, L.; Goh, B.C.; et al. Targeting transcription factor STAT3 for cancer prevention and therapy. Pharmacol. Ther. 2016, 162, 86-97. [CrossRef] [PubMed]

42. Hynes, N.E.; Lane, H.A. ERBB receptors and cancer: The complexity of targeted inhibitors. Nat. Rev. Cancer 2005, 5, 341-354. [CrossRef] [PubMed]

43. Bode, J.G.; Ehlting, C.; Haussinger, D. The macrophage response towards LPS and its control through the p38(MAPK)-STAT3 axis. Cell Signal. 2012, 24, 1185-1194. [CrossRef] [PubMed]

44. Wierenga, A.T.; Vogelzang, I.; Eggen, B.J.; Vellenga, E. Erythropoietin-induced serine 727 phosphorylation of STAT3 in erythroid cells is mediated by a MEK-, ERK-, and MSK1-dependent pathway. Exp. Hematol. 2003, 31, 398-405. [CrossRef]

45. Kane, L.P.; Mollenauer, M.N.; Xu, Z.; Turck, C.W.; Weiss, A. Akt-dependent phosphorylation specifically regulates Cot induction of NF-kappa B-dependent transcription. Mol. Cell. Biol. 2002, 22, 5962-5974. [CrossRef] [PubMed]

46. Gao, N.; Zhang, Z.; Jiang, B.H.; Shi, X. Role of PI3K/AKT/mTOR signaling in the cell cycle progression of human prostate cancer. Biochem. Biophys. Res. Commun. 2003, 310, 1124-1132. [CrossRef] [PubMed]

47. Downward, J. Targeting RAS signalling pathways in cancer therapy. Nat. Rev. Cancer 2003, 3, 11-22. [CrossRef] [PubMed]

48. Vogelstein, B.; Lane, D.; Levine, A.J. Surfing the p53 network. Nature 2000, 408, 307-310. [CrossRef] [PubMed]

49. Michael, D.; Oren, M. The p53 and Mdm2 families in cancer. Curr. Opin. Genet. Dev. 2002, 12, 53-59. [CrossRef]

50. Ito, K.; Nakazato, T.; Yamato, K.; Miyakawa, Y.; Yamada, T.; Hozumi, N.; Segawa, K.; Ikeda, Y.; Kizaki, M. Induction of apoptosis in leukemic cells by homovanillic acid derivative, capsaicin, through oxidative stress: Implication of phosphorylation of p53 at Ser-15 residue by reactive oxygen species. Cancer Res. 2004, 64, 1071-1078. [CrossRef] [PubMed]

(C) 2017 by the authors. Licensee MDPI, Basel, Switzerland. This article is an open access article distributed under the terms and conditions of the Creative Commons Attribution (CC BY) license (http://creativecommons.org/licenses/by/4.0/). 